\title{
Associations Among Disseminated Intravascular Coagulation, Thrombocytopenia Cytokines/ Chemokines and Genetic Polymorphisms of Toll-Like Receptor 2/4 in Chinese Patients with Sepsis
}

\author{
Shaowei Jiang' \\ Jing $\mathrm{Ma}^{2}$ \\ Shan $\mathrm{Ye}^{3}$ \\ Connor Meaney ${ }^{4}$ \\ Timothy Evan Moore ${ }^{4}$ \\ Shuming Pan' \\ Chengjin $\mathrm{Gao} \mathbb{D}^{\prime}$ \\ 'Department of Emergency, Xinhua \\ Hospital Affiliated to Shanghai Jiao Tong \\ University School of Medicine, Shanghai, \\ 200092, People's Republic of China; \\ ${ }^{2}$ Department of Pharmacy, Xinhua \\ Hospital Affiliated to Shanghai Jiao Tong \\ University School of Medicine, Shanghai, \\ 200092, People's Republic of China; \\ ${ }^{3}$ Department of Internal Medicine, \\ Shanghai Songnan Community Health \\ Service Center, Shanghai, 200092. \\ People's Republic of China; ${ }^{4}$ Division of \\ Rheumatology, Department of Internal \\ Medicine, University of Utah School of \\ Medicine, Salt Lake City, Utah, \\ 84132 , USA
}

Correspondence: Chengjin Gao; Shuming Pan

Email gaochengjin@xinhuamed.com.cn; panshuming@xinhuamed.com.cn

\begin{abstract}
Objectives(s): Toll-like receptors (TLRs) on platelets have been extensively studied. Both TLR2 and TLR4 have been shown to augment platelet activation and alter its function from a hemostatic regulator to an immune sentinel. However, few studies have investigated the relationship between genetic polymorphisms in TLR2, TLR4 and platelets. We investigated whether genetic polymorphisms of TLR2 and TLR4 were related to thrombocytopenia and coagulation failure in Chinese patients with sepsis.
\end{abstract}

Basic Methods: Adult Chinese patients with sepsis in the intensive care unit of a university medical center were monitored for up to 28 days. Thrombocytopenia and disseminated intravascular coagulation (DIC), diagnosed using Japanese Association for Acute Medicine (JAAM) criteria, were observed as the primary outcomes. Single-nucleotide polymorphisms (SNPs) in TLR2 (rs111200466, rs5743708) and TLR4 (rs11536889, rs145801336, rs11536896, rs7869402) in patients with sepsis were detected by polymerase chain reaction. The data were analyzed using chi-square and rank sum tests.

Results: The genotype of TLR2 (rs111200466) (Del/Del) was associated with the initial DIC. The genotype of TLR4 (rs11536889) (C/C\&C/G) was associated with initial DIC, DIC onset during hospitalization and platelet counts. Furthermore, both DIC and platelet counts were associated with cytokines and chemokines, especially the IL10.

Conclusion: Our results demonstrate that in Chinese sepsis patients, the rs111200466 SNP in TLR2 and rs11536889 SNP in TLR4 are associated with thrombocytopenia and DIC, with potential effects on the TLR4 pathways of platelets.

Keywords: sepsis, disseminated intravascular coagulation, TLR2/4, genetic polymorphisms

\section{Introduction}

Sepsis is a life-threatening organ dysfunction that results from an exaggerated host immune response to disseminate infection. ${ }^{1}$ Despite improvements in treatment strategies, sepsis remains a leading cause of death in critically ill patients worldwide. $^{2}$ Low platelet number, known as thrombocytopenia, is common in infectious diseases (also sometimes referred to as sepsis). ${ }^{3}$ The most significant features of sepsis are thrombocytopenia and disseminated intravascular coagulation (DIC). ${ }^{4}$ Patients with severe COVID-19 present with hemostatic abnormalities that mimic DIC associated with sepsis with the major difference being an increased risk of thrombosis rather than bleeding. ${ }^{5}$ Thrombocytopenia is observed in some patients with COVID-19 and is correlated with mortality. ${ }^{6}$ 
Thrombocytopenia is a common finding in critically ill patients, increasing sepsis-related morbidity and mortality ${ }^{7}$ this condition is independently associated with a more disturbed host response in critically ill patients with sepsis. ${ }^{4}$ Although the role of platelets in blood clotting is well known, platelets mediate key aspects of inflammatory and immune processes. ${ }^{3}$ For example, platelets limit bacterial growth and dissemination in experimental sepsis, ${ }^{8}$ as well as influence leukocyte recruitment and functions, cytokine responses, vascular endothelium activity and the coagulation system. ${ }^{9}$ Platelets detect foreign pathogens, including viruses, through pattern recognition receptors (eg, Toll-like receptors, TLRs), ${ }^{10}$ and coordinate with PMNs (polymorph nuclear neutrophils) to release neutrophil extracellular traps (NETs) through chemokine and coagulation factor signaling. ${ }^{11}$ Coagulation abnormalities are common in severe sepsis. Patients with systemic infection often develop DIC, a condition involving a systemic activation of the clotting cascade, leading to fibrin deposition and microthrombus formation throughout the capillaries and, resulting in pulmonary embolism, multiorgan dysfunction syndrome, and death. ${ }^{12}$

TLRs are hallmarks of innate immunity and signal transducers of responsive cells to induce the expression of genes that produce defense proteins. TLRs on platelets have been extensively studied., ${ }^{3,13-15}$ Human platelets express TLRs $1-10$, and mouse platelets express TLRs $1-8 .{ }^{16}$ These TLRs are functional on platelets, as stimulation of platelets with lipopolysaccharide (LPS) or other TLR-ligands caused thrombocytopenia and the production of tumor necrosis factor- $\alpha$ and other potential mediators of inflammation (ie thromboxane, tissue factor, proinflammatory cytokines, etc.) under in vivo conditions. ${ }^{17}$ Both TLR2 and TLR4 augment platelet activation and alter their function from hemostatic regulators to immune sentinels. TLR4 is the most abundantly expressed TLR in platelets, and its expression in these cells may significantly modulate LPS-induced thrombocytopenia and TNF- $\alpha$ production in vivo. ${ }^{18}$ It has been reported that $T L R s$ presented on platelets could be the linker between DIC and sepsis. ${ }^{19}$

Immunopolymorphism is important in the resistance or susceptibility of the host to an infectious disease. Protein(s) encoded by TLR gene(s) are immensely important due to their ability to recognize different types of pathogen-associated molecular patterns (PAMP). ${ }^{20}$ Hyperactivation or inactivation of TLRs is mainly caused by mutations in TLR gene that affect the normal functioning of these receptors. ${ }^{21}$ Intriguingly, the frequency of TLR polymorphisms and their functions vary in different geographic regions, with natural selection considered as the principal driving force behind such diversity. ${ }^{20}$ Two single-nucleotide polymorphisms (SNPs) in the promoter region of TLR4 can lead to hyporesponsiveness to LPS. ${ }^{22}$ Strikingly, it has been reported that platelets can express functional $T L R 4,{ }^{23}$ and adults with $T L R 4$ variant genotypes show significant differences in platelet function. ${ }^{24}$ However, few studies have focused on the relationship among TLRs variants, the platelets status, and DIC during sepsis. In this study, we investigated the association among TLR2 and TLR4 polymorphisms, thrombocytopenia and coagulation failure in Chinese patients with sepsis.

\section{Materials and Methods Patients}

The clinical characteristics of the study subjects are shown in Table 1. A total of 181 patients (68 female and 113 male) aged $18-80$ years (average age $58.71 \pm 10.65$ ) with sepsis were recruited between November 2016 and August 2018 in the intensive care unit (ICU) of Xinhua Hospital, affiliated to Shanghai Jiao Tong University School of Medicine, Shanghai, PR, China. The study was approved by the Xinhua Hospital Ethics committee affiliated with Shanghai Jiao Tong University School of Medicine, China (Ethics number: XHEC-D-2020-010), and conformed to the Declaration of Helsinki ethical principles (Seoul, 2008). Written informed consent was obtained from either the patients or their legal representatives.

\section{Inclusion Criteria}

The inclusion criteria were in accordance with the Assessment of Clinical Criteria for Sepsis. ${ }^{1}$ In the Third International Consensus Definitions for Sepsis and Septic Shock (Sepsis-3), ${ }^{1}$ septic shock was defined as circulatory, cellular, and metabolic abnormalities in patients with sepsis, presenting as fluid refractory hypotension requiring vasopressor therapy with associated tissue hypoperfusion (lactate $>2 \mathrm{mmol} / \mathrm{L}$ ). Patients were enrolled within $24 \mathrm{~h}$ of developing these criteria.

\section{Exclusion Criteria}

The patient exclusion criteria were as follows: age younger than 18 years; pregnancy or nursing an infant; 
Table I Baseline Clinical Characteristics of the Population

\begin{tabular}{|c|c|}
\hline Demographics & $\begin{array}{l}\text { Value (n, \% Unless } \\
\text { Otherwise Specified) }\end{array}$ \\
\hline Age (years), mean $\pm S D$ & $58.71 \pm 10.65(18-80)$ \\
\hline Sex (male: female), no. (\%) & (I I3:68) (62.4\%:37.6\%) \\
\hline Septic shock, \% & $42(23.2 \%)$ \\
\hline Use of vasopressors & $33(18.2 \%)$ \\
\hline Initial SOFA score, mean \pm SD & $5.33 \pm 3.29(2-15)$ \\
\hline Initial APACHE II score, mean \pm SD & $14.44 \pm 8.5(0-42)$ \\
\hline Comorbidities, \% & \\
\hline Hypertension & 74 (40.9\%) \\
\hline COPD & $6(3.3 \%)$ \\
\hline Chronic heart failure & $33(18.2 \%)$ \\
\hline Diabetes mellitus & $53(29.3 \%)$ \\
\hline Cancer history & II (6.I\%) \\
\hline Stroke history & $30(16.6 \%)$ \\
\hline Site of infection, \% & \\
\hline Respiratory, n (\%) & $84(46.4 \%)$ \\
\hline Abdominal, n (\%) & $66(36.6 \%)$ \\
\hline Urinary, n (\%) & $26(14.4 \%)$ \\
\hline Bloodstream, n (\%) & $3(1.7 \%)$ \\
\hline Neurological, n (\%) & $\mathrm{I}(0.6 \%)$ \\
\hline Soft tissue, n (\%) & I (0.6\%) \\
\hline $\begin{array}{l}\text { Initial DIC score according to the } \\
\text { JAAM criteria, mean } \pm \text { SD }\end{array}$ & $2.17 \pm 2.05(0-8)$ \\
\hline $\begin{array}{l}\text { Initial DIC onset according to the } \\
\text { JAAM criteria }\end{array}$ & $40(22.1 \%)$ \\
\hline $\begin{array}{l}\text { Maximum DIC score according to the } \\
\text { JAAM criteria, mean } \pm S D\end{array}$ & $3.01 \pm 2.38(0-8)$ \\
\hline $\begin{array}{l}\text { DIC onset according to the JAAM } \\
\text { criteria }\end{array}$ & $70(38.7 \%)$ \\
\hline $\begin{array}{l}\text { Initial DIC score according to the ISTH } \\
\text { criteria, mean } \pm S D\end{array}$ & $2.44 \pm 1.39(0-8)$ \\
\hline $\begin{array}{l}\text { Initial DIC onset according to the ISTH } \\
\text { criteria }\end{array}$ & $9(5 \%)$ \\
\hline $\begin{array}{l}\text { Maximum DIC score according to the } \\
\text { ISTH criteria, mean } \pm \text { SD }\end{array}$ & $3.01 \pm 1.48(0-8)$ \\
\hline $\begin{array}{l}\text { DIC onset according to the ISTH } \\
\text { criteria }\end{array}$ & $20(11 \%)$ \\
\hline 28-day mortality & $32(17.7 \%)$ \\
\hline
\end{tabular}

Abbreviations: Initial SOFA score, highest Sequential Organ Failure Assessment scores within the first 24 hours after ICU admission; initial APACHE II score, highest Acute Physiology and Chronic Health Evaluation II score within the first 24 hours after ICU admission; COPD, chronic obstructive pulmonary disease; DIC, disseminated intravascular coagulation; JAAM, Japanese Association for Acute Medicine; ISTH, International Society on Thrombosis and Haemostasis. hematopoietic malignancy; liver cirrhosis (Child-Pugh class C); administration of immunosuppressive therapy (eg, cyclosporine or azathioprine) or cancer-related chemotherapy or radiotherapy; human immunodeficiency virus infection or end-stage process; morbidity and death considered as imminent; participation in any other investigational study (drug or device); or patient unwilling or unable to be fully evaluated during the study period.

\section{DNA Extraction and Genotyping}

A 5-mL venous blood sample was collected in EDTA sterile tubes from each patient and stored at $-80^{\circ} \mathrm{C}$ until the DNA was extracted from whole blood samples using kits (AxyPrep, Axygen Scientific, Union City, CA, USA) according to the manufacturer's instructions. The variant information for TLR2 and TLR4 are available on the NCBI website (https://www.ncbi.nlm.nih.gov/snp/). The polymorphisms relevant to this study were rs111200466 and rs5743708 in TLR2, and rs11536889, rs145801336, rs11536896, and rs7869402 in TLR4. These variants were genotyped by Sanger sequencing. First, the exons of TLR2 and TLR4 were cloned using KOD enzyme mix (TOYOBO, Osaka, Japan) on an ABI Veriti 96 thermal cycler (Applied Biosystems, Foster City, CA, USA). The primers used for cloning are listed in Supplementary Table 1. DNA sequencing was performed on an ABI 3730XL (Applied Biosystems), and the sequencing primers are shown on Supplementary Table 1.

\section{Data Collection}

The following parameters and information of each patient were recorded at the time of admission to the ICU: sex, age, diabetes mellitus, hypertension, chronic heart failure, chronic obstructive pulmonary disease, cancer history, stroke history, and infection site. The need for vasopressor administration and the ICU duration were also recorded. As part of routine clinical practice and laboratory monitoring, assessments and blood sampling were performed daily after admission to the ICU. D-dimer and fibrinogen degradation products were detected by immunoturbidimetry, prothrombin time, activated partial thromboplastin time, thrombin time and fibrinogen were detected by coagulase. The Japanese Association for Acute Medicine (JAAM) DIC criteria ${ }^{25}$ and International Society on Thrombosis and Hemostasis (ISTH) criteria for overt DIC (ISTH DIC $)^{26}$ were used to assess patients with sepsis. Initial DIC scores were defined as the highest DIC scores within 
the first $24 \mathrm{~h}$ after ICU admission of the patients; maximum DIC scores were defined as the maximum DIC scores measured during hospitalization within 28 days from admission. Initial DIC onset was defined as DIC occurring within the first $24 \mathrm{~h}$ after ICU admission of the patients; DIC onset was defined as DIC occurring during hospitalization within 28 days of admission. DIC was managed with a combination of anticoagulants and plasma and/or platelet transfusion. The Sequential Organ Failure Assessment (SOFA) ${ }^{27}$ and Acute Physiology and Chronic Health Evaluation (APACHE II) ${ }^{28}$ scores were evaluated at the onset of sepsis. Follow-up evaluations were performed for up to 28 days. To determine the immunological status of patients with sepsis, human leukocyte antigen, the percentage of CD14+ human leukocyte antigen-DR+, $\mathrm{CD} 3, \mathrm{CD} 4$, and $\mathrm{CD} 8$ counts, percentages of $\mathrm{CD} 3$ and $\mathrm{CD} 8$, and ratio of $\mathrm{CD} 4$ to $\mathrm{CD} 8$ were analyzed. We also measured the serum levels of interleukin (IL)-1 $\beta$, IL-2R, IL-6, IL-8, IL-10, tumor necrosis factor- $\alpha$, and interferon- $\gamma$ at admission.

The lowest platelet count within the first $24 \mathrm{~h}$ after ICU admission and minimum platelet count during hospitalization was used to stratify patients into groups with very low platelet counts $\left(<50 \times 10^{9} / \mathrm{L}\right)$, intermediate-low platelet counts $\left(50 \times 10^{9}-99 \times 10^{9} / \mathrm{L}\right)$, low platelet counts $(100 \times$ $\left.10^{9}-149 \times 10^{9} / \mathrm{L}\right)$, or normal platelet counts $\left(150 \times 10^{9}-\right.$ $\left.399 \times 10^{9} / \mathrm{L}\right)$, with limits based on previous studies. ${ }^{4}$

The initial leukocyte count, neutrophil granulocyte count, and neutrophil-lymphocyte ratio (NLR) were recorded. The NLR is a rapid and simple assessment reflecting systemic inflammation and stress, and a high NLR indicates an unfavorable prognosis in patients with sepsis. ${ }^{29}$ The maximum leukocyte count, neutrophil granulocyte count, NLR, initial platelet count, and minimum platelet count during hospitalization were assessed. Neutrophils are activated when sepsis occurs, causing a series of chemokine changes. To further identify the chemokines associated with neutrophils, including CXCL1 (GRO $\alpha$ ), CXCL5 (ENA-78), CXCL8 (IL-8), CXCL9 (MIG), CXCL10 (IP-10), CXCL11 (I-TAC), CCL2 (MCP-1), CCL3 (MIP-1 $\alpha)$, CCL4 (MIP-1 $\beta$ ), CCL5 (RANTES), CCL11 (Eotaxin), CCL17 (TARC), and CCL20 (MIP-3 $\alpha$ ), we chose the serum sample with the highest NLR during hospitalization, which indicated substantially more serious septic conditions.

Proinflammatory chemokines in the serum were detected using the Proinflammatory Chemokine Panel (13plex) (cat\#740003, BioLegend, San Diego, CA, USA) according to the manufacturer's instructions, and analyzed using a flow cytometer (CytoFLEX S, Beckman, Brea, CA, USA). PAI-1 antigen was assayed using a specific enzyme-linked immunosorbent assay (Human PAI-1 ELISA Kit, cat\# 1818272A, Life Technologies, Carlsbad, CA, USA), according to the manufacturer's instructions.

\section{Statistical Analysis}

Quantitative variables were described as the mean and standard deviation and qualitative data as a number and percentage. Univariate logistic regression and multivariate (stepwise logistic regression, with enter and removal limits set at 0.10 and factors significant at $p=0.05$ included) analyses were performed to identify the factors associated with initial DIC and DIC onset during hospitalization according to JAAM criteria, minimum platelet count during hospitalization (100 versus $\geq 100$ ), and genotypes of TLR2 (rs111200466, rs5743708) and TLR4 (rs11536889, rs145801336, rs11536896, rs7869402). A $p$ value $<0.05$ was considered as significant. The odds ratio (OR) and $95 \%$ confidence intervals (CIs) were calculated. Statistical regression tests were performed using SAS Version 9.4 software (SAS, Inc., Cary, NC, USA).

\section{Results}

\section{Baseline Characteristics}

The severity of sepsis were sepsis $(n=137)$ and septic shock $(\mathrm{n}=44)$. At baseline, the patient disease severity SOFA and APACHE II scores were $5.33 \pm 3.29(2-15)$ and $14.44 \pm 8.5(0-42)$, respectively (Table 1$)$. Initial and maximum DIC scores by JAAM criteria were $2.17 \pm$ $2.05(0-8)$ and $3.01 \pm 2.38(0-8)$, whereas the scores by ISTH criteria were $2.44 \pm 1.39(0-8)$ and $3.01 \pm 1.48(0-$ 8 ), respectively. Of all the patients enrolled in the study, 32 $(17.7 \%)$ died within 28 days. We also recorded comorbidities, which were mainly hypertension, chronic heart failure, chronic obstructive pulmonary disease, diabetes mellitus, cancer history, and stroke history (Table 1).

\section{Prognostic Factors for DIC Onset by JAAM Criteria During Hospitalization}

DIC is an important prognostic factor for sepsis death. We drew the 28-day survival plots for patients with and without DIC onset as per JAAM criteria during hospitalization. The overall 28-day survival rate in patients with DIC onset was significantly lower than that in patients without DIC 
onset ( $p=0.0095)$. The survival plot for DIC onset as per JAAM criteria during hospitalization is shown in Figure 1.

Eight independent associated factors for DIC onset, according to JAAM criteria, were identified during hospitalization by univariate and multivariate logistic regression analysis. These factors were increased initial APACHE II scores (OR 1.23; 95\% CI 1.05-1.44), death (OR 168.55; 95\% CI 2.94-9670.65), very low platelet counts (OR 211.76; 95\% CI 1.30-34,618.90) and intermediate-low platelet counts during hospitalization (OR 206.80; 95\% CI 5.24-8168.27), decreased CD8 counts (OR 0.99; 95\% CI 0.97-1.00), increased serum levels of IL-1 $\beta$ (OR 16.18; 95\% CI 0.66-397.99), PAI-1 (OR 33.07; 95\% CI 2.17503.23), and CCL20 (OR 1.01; 95\% CI 1.00-1.02) (Table 2). The additional factors associated with DIC onset during hospitalization in univariate analysis included shock, intermediate-low platelet counts on admission, initial NLR, maximum white blood cell counts, maximum neutrophil counts and the maximum percentage of neutrophils during hospitalization, lower CD3 and CD4 counts, increased serum levels of IL-2R, IL-6, IL-10, CXCL1, CXCL9, CCL2, and CCL3, decreased serum level of CCL5, increased initial and maximum serum levels of CXCL8, higher initial SOFA and DIC scores by JAAM criteria, higher DIC scores by ISTH criteria during hospitalization, and genotype of TLR4 (rs11536889) (C/C\&C/ G) (Table 2).

\section{Prognostic Factors for Initial DIC Onset by JAAM Criteria}

The initial DIC is another important DIC indicator, which reflects the severity of infection onset. Intermediate-low (OR 13.10; 95\% CI 1.10-156.42) and low platelet counts during hospitalization (OR 1.55; 95\% CI 0.10-23.19), as well as higher DIC scores (OR 1.72; 95\% CI 1.15-2.58) during hospitalization, were significantly associated with

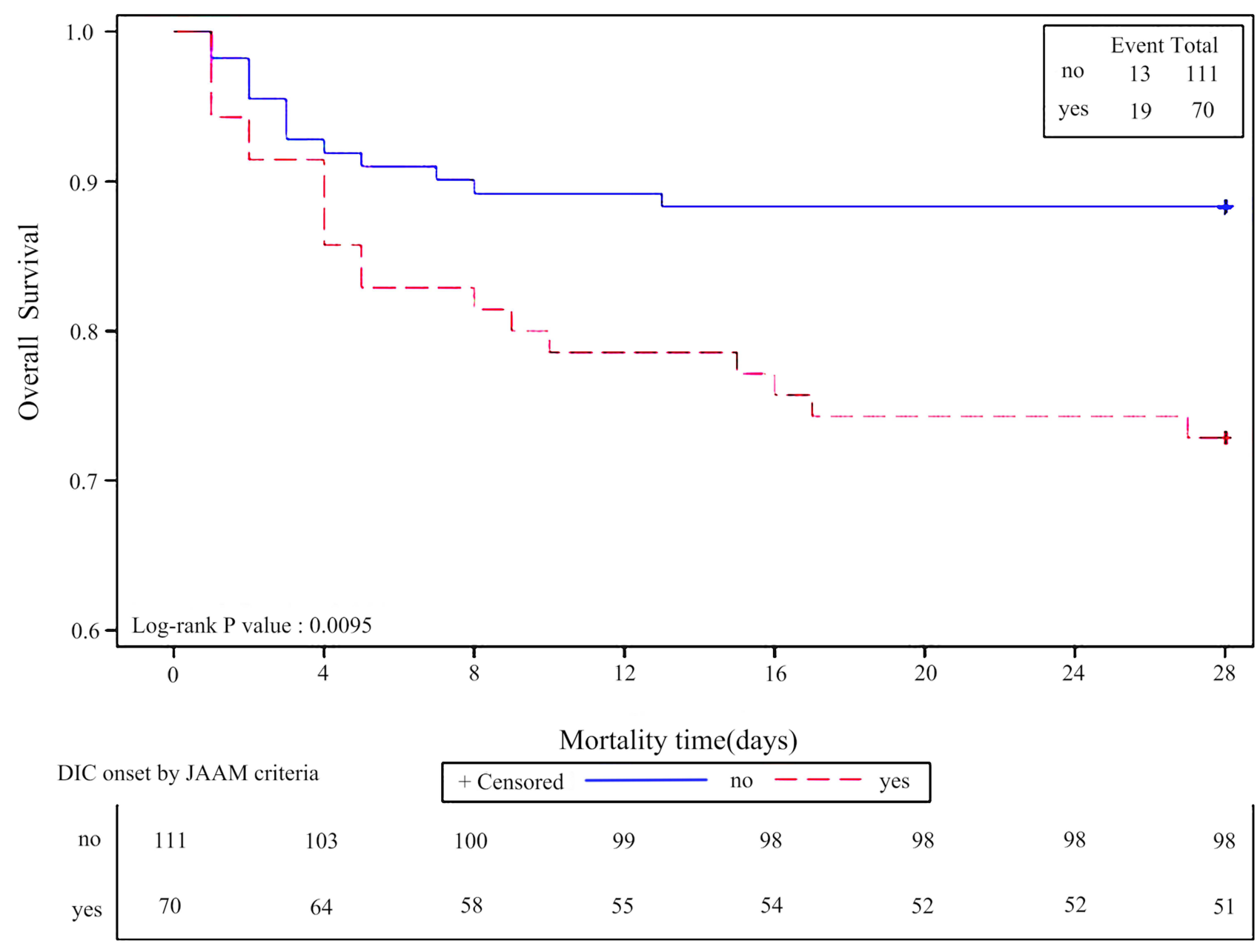

Figure I Survival plot for DIC onset by JAAM criteria. The 28-days survival plots for patients with and without DIC onset by JAAM criteria during hospitalization. The overall 28-days survival rate in patients with DIC onset by JAAM criteria was significantly lower than patients without $D I C$ onset by JAAM criteria $(P=0.0095)$. 
Table 2 Prognostic Factors for DIC Onset by JAAM Criteria During Hospitalization

\begin{tabular}{|c|c|c|c|c|}
\hline Factors & No & Yes & $\begin{array}{c}\text { Univariate } \\
\text { Analysis OR } \\
\text { (95\% Cl) }\end{array}$ & $\begin{array}{c}\text { Multivariate } \\
\text { Analysis OR } \\
(95 \% \mathrm{Cl})^{*}\end{array}$ \\
\hline \multicolumn{5}{|l|}{ Shock } \\
\hline No & 95 (85.59) & $44(62.86)$ & 1.00 & \\
\hline Yes & $16(14.4 I)$ & $26(37.14)$ & $3.52(1.71,7.19)$ & \\
\hline \multicolumn{5}{|l|}{ Death } \\
\hline No & 98 (88.29) & $51(72.86)$ & 1.00 & 1.00 \\
\hline Yes & $13(|1.7|)$ & $19(27.14)$ & $2.81(1.28,6.14)$ & I $68.55(2.94,9670.65)$ \\
\hline \multicolumn{5}{|l|}{ Platelet count on admission $\left(145 \sim 400 \times 10^{9} / \mathrm{L}\right)$} \\
\hline$<50$ & $0(0.00)$ & $5(7.14)$ & - & \\
\hline $50-100$ & $10(9.01)$ & $31(44.29)$ & II.43 (4.64,28.17) & \\
\hline $100-150$ & $42(37.84)$ & $18(25.7 \mid)$ & $1.58(0.72,3.45)$ & \\
\hline$>150$ & $59(53.15)$ & $16(22.86)$ & 1.00 & \\
\hline \multicolumn{5}{|l|}{ Platelet count, minimum $\left(145 \sim 400 \times 10^{9} / \mathrm{L}\right)$} \\
\hline$<50$ & I $(0.90)$ & $19(27.14)$ & $\begin{array}{c}118.65 \\
(13.90,1012.73)\end{array}$ & $\begin{array}{c}211.76 \\
(1.30,346 \mid 8.90)\end{array}$ \\
\hline $50-100$ & $13(11.71)$ & 31 (44.29) & I $4.90(5.55,40.04)$ & $206.80(5.24,8168.27)$ \\
\hline $100-150$ & $47(42.34)$ & $12(17.14)$ & $1.60(0.60,4.25)$ & $0.39(0.02,6.30)$ \\
\hline$>150$ & $50(45.05)$ & $8(11.43)$ & 1.00 & 1.00 \\
\hline NLR on admission, mean \pm SD & $17.09 \pm 14.62$ & $24.28 \pm 26.80$ & $1.02(1.00,1.04)$ & \\
\hline $\mathrm{WBC} \max$, mean $\pm \mathrm{SD}\left(4 \sim 10 \times 10^{9} / \mathrm{L}\right)$ & $13.8 \mid \pm 6.28$ & $16.64 \pm 8.19$ & $1.06(1.01,1.10)$ & \\
\hline Neutrophil max, mean \pm SD $\left(1.4 \sim 6.5 \times 10^{9} / \mathrm{L}\right)$ & $12.06 \pm 5.94$ & $14.88 \pm 7.63$ & $1.06(1.02,1.12)$ & \\
\hline Neutrophil percentage max (\%), mean \pm SD & $86.03 \pm 8.27$ & $90.15 \pm 6.32$ & $1.08(1.03,1.14)$ & \\
\hline NLR max, mean \pm SD & $22.99 \pm 19.16$ & $34.41 \pm 30.54$ & $1.02(1.01,1.03)$ & \\
\hline $\mathrm{CD} 3$, mean $\pm \mathrm{SD}(|| 4|\sim| 880 / \mu \mathrm{L})$ & $670.08 \pm 345.41$ & $408.30 \pm 258.75$ & $1.00(1.00,1.00)$ & \\
\hline $\mathrm{CD} 4$, mean $\pm \mathrm{SD}(478 \sim 1072 / \mu \mathrm{L})$ & $409.11 \pm 232.80$ & $238.40 \pm 175.72$ & $1.00(1.00,1.00)$ & \\
\hline CD8, mean $\pm \mathrm{SD}(393 \sim 742 / \mu \mathrm{L})$ & $233.34 \pm 150.37$ & $148.36 \pm 104.32$ & $1.00(1.00,1.00)$ & $0.99(0.97,1.00)$ \\
\hline \multicolumn{5}{|l|}{ IL-I $\beta$ on admission $(\mathrm{pg} / \mathrm{mL})$} \\
\hline$<5$ & $59(93.65)$ & $24(75.00)$ & 1.00 & 1.00 \\
\hline$\geq 5$ & $4(6.35)$ & $8(25.00)$ & $4.92(1.35,17.87)$ & $16.18(0.66,397.99)$ \\
\hline $\mathrm{IL}-2 \mathrm{R}$ on admission, mean $\pm \mathrm{SD}(\mathrm{U} / \mathrm{mL})$ & $1003.6 \pm 719.28$ & $2082.2 \pm 11129.6$ & $1.00(1.00,1.00)$ & \\
\hline IL- 6 on admission, mean $\pm \mathrm{SD}(\mathrm{pg} / \mathrm{mL})$ & $89.33 \pm 171.61$ & $216.20 \pm 293.65$ & $1.00(1.00,1.00)$ & \\
\hline $\mathrm{IL}-8$ on admission, mean $\pm \mathrm{SD}(\mathrm{pg} / \mathrm{mL})$ & $35.49 \pm 31.60$ & $371.05 \pm 1183.5$ & $1.02(1.00,1.03)$ & \\
\hline \multicolumn{5}{|l|}{ IL-I0 on admission $(\mathrm{pg} / \mathrm{mL})$} \\
\hline$<9.1$ & $51(80.95)$ & $12(37.50)$ & 1.00 & \\
\hline$\geq 9.1$ & $12(19.05)$ & $20(62.50)$ & $7.08(2.73, \mid 8.36)$ & \\
\hline \multicolumn{5}{|l|}{ PAI-I (ng/mL) } \\
\hline$\leq 4363$ & 75 (67.57) & $31(44.29)$ & 1.00 & 1.00 \\
\hline$>4363$ & $36(32.43)$ & $39(55.7 I)$ & $2.62(1.41,4.86)$ & $33.07(2.17,503.23)$ \\
\hline $\mathrm{CXCLI}$, mean $\pm \mathrm{SD}(\mathrm{pg} / \mathrm{mL})$ & $149.78 \pm 188.97$ & $269.88 \pm 479.29$ & $1.00(1.00,1.00)$ & \\
\hline $\mathrm{CXCL} 8$, mean $\pm \mathrm{SD}(\mathrm{pg} / \mathrm{mL})$ & $159.59 \pm 276.33$ & $306.23 \pm 381.64$ & $1.00(1.00,1.00)$ & \\
\hline $\mathrm{CXCL}$, mean $\pm \mathrm{SD}(\mathrm{pg} / \mathrm{mL})$ & $56.55 \pm 91.33$ & $88.85 \pm 103.77$ & $1.00(1.00,1.01)$ & \\
\hline $\mathrm{CCL} 2$, mean $\pm \mathrm{SD}(\mathrm{pg} / \mathrm{mL})$ & $743.17 \pm 999.43$ & $1580.5 \pm 1621.7$ & $1.00(1.00,1.00)$ & \\
\hline $\mathrm{CCL} 3$, mean $\pm \mathrm{SD}(\mathrm{pg} / \mathrm{mL})$ & $17.34 \pm 22.48$ & $33.70 \pm 44.20$ & $1.02(1.00,1.03)$ & \\
\hline $\mathrm{CCL} 5$, mean $\pm \mathrm{SD}(\mathrm{pg} / \mathrm{mL})$ & $2362.7 \pm 1457.0$ & $1659.1 \pm 1212.0$ & $1.00(1.00,1.00)$ & \\
\hline $\mathrm{CCL} 20$, mean $\pm \mathrm{SD}(\mathrm{pg} / \mathrm{mL})$ & $98.43 \pm 160.90$ & $204.65 \pm 216.55$ & $1.00(1.00,1.00)$ & $1.01(1.00,1.02)$ \\
\hline
\end{tabular}

(Continued) 
Table 2 (Continued).

\begin{tabular}{|c|c|c|c|c|}
\hline Factors & No & Yes & $\begin{array}{c}\text { Univariate } \\
\text { Analysis OR } \\
\text { (95\% Cl) }\end{array}$ & $\begin{array}{c}\text { Multivariate } \\
\text { Analysis OR } \\
(95 \% \mathrm{Cl})^{*}\end{array}$ \\
\hline Initial APACHE II, mean \pm SD & $12.73 \pm 7.90$ & $17.34 \pm 8.58$ & $1.07(1.03,1.11)$ & $1.23(1.05,1.44)$ \\
\hline Initial SOFA, mean \pm SD & $4.46 \pm 2.88$ & $6.70 \pm 3.46$ & $1.24(1.12,1.38)$ & \\
\hline Initial DIC scores by JAAM criteria, mean \pm SD & $1.03 \pm 0.94$ & $3.97 \pm 2.05$ & $4.21(2.78,6.37)$ & \\
\hline Maximum DIC scores by ISTH criteria, mean \pm SD & $2.20 \pm 0.99$ & $4.29 \pm 1.21$ & $13.58(6.29,29.32)$ & \\
\hline \multicolumn{5}{|l|}{ TLR4(rs I I536889) } \\
\hline $\mathrm{G} / \mathrm{G}$ & $73(66.97)$ & $34(48.57)$ & 1.00 & \\
\hline $\mathrm{C} / \mathrm{C} \& \mathrm{C} / \mathrm{G}$ & $36(33.03)$ & $36(51.43)$ & $2.15(1.16,3.97)$ & \\
\hline
\end{tabular}

Notes: *Univariate logistic regression and multivariate analyses were performed to determine the factors associated with DIC onset by JAAM criteria during hospitalization. A p value $<0.05$ was considered significant. Odds ratios $(\mathrm{ORs})$ and their $95 \%$ confidence intervals were calculated.

initial DIC onset in both univariate and multivariate regression analysis (Table 3 ). The additional factors associated with initial DIC onset during hospitalization in univariate analysis included shock, death, platelet counts on admission, maximum percentage of neutrophil during hospitalization, lower CD3 and CD4 counts, increased serum levels of IL-2R, IFN- $\gamma$, IL-10, CXCL8, CXCL9, CXCL10, CCL2, CCL3, CCL4, and CCL20, decreased serum levels of CCL5, higher initial SOFA and DIC scores by JAAM criteria, higher DIC scores by ISTH criteria and DIC onset by ISTH criteria during hospitalization, and genotypes of TLR2 (rs111200466) (Del/Del) and TLR4 (rs11536889) (C/C\&C/G) (Table 3).

\section{Potential Factors Predictive of Minimum Platelet Count During Hospitalization}

A low platelet level indicates severe sepsis and is associated with a high death rate. We analyzed the prognostic factors related to the minimum platelet count. In multivariate analysis, decreased initial white blood cell counts (OR 1.23; 95\% CI 1.05-1.45), increased serum levels of IL-2R (OR 1.00; 95\% CI 1.00-1.00), IL-8 (OR 0.97; 95\% CI 0.94-0.99) and CCL20 (OR 1.01; 95\% CI 1.00-1.02), and higher DIC scores as per ISTH criteria (OR 0.13; 95\% CI 0.04-0.44) were associated with a minimum platelet count $<100$ during hospitalization (Table 4). Further, during hospitalization, shock, decreased initial neutrophil and platelet counts, decreased percentage of CD4, decreased counts of CD3 and CD4, increased serum levels of IL-6, IL-10, PAI-1, CXCL8, CCL2, and CCL3, decreased serum levels of CCL5, higher initial SOFA scores, higher initial and maximum DIC scores as per JAAM criteria, higher initial and maximum DIC onset as per JAAM criteria, higher DIC onset as per ISTH criteria, and the genotypes of TLR4 (rs11536889) $(\mathrm{C} / \mathrm{C} \& \mathrm{C} / \mathrm{G})$ were associated with a minimum platelet count $<100$ in univariate analysis.

\section{Prognostic Factors for TLR2/4 SNP}

TLRs regulate platelet activity, as reported in some studies, and are associated with DIC and platelet counts during sepsis. Therefore, we analyzed which clinical factors were related to TLR4 or TLR2 polymorphism. Intermediate-low platelet counts on admission (OR 2.93; 95\% CI 1.29-6.66) and genotype of TLR4 (rs7869402) (85bpC/T) (OR 0.08; 95\% CI 0.01-0.60) were associated with TLR4 (rs11536889) $(\mathrm{C} / \mathrm{C} \& \mathrm{C} / \mathrm{G})$ in multivariate analysis (Table 5). Very low platelet counts during hospitalization, higher initial and maximum DIC scores by JAAM criteria, higher initial and maximum DIC onset by JAAM criteria, and higher initial and maximum DIC onset by ISTH criteria were associated with TLR4 (rs11536889) $(\mathrm{C} / \mathrm{C} \& \mathrm{C} / \mathrm{G})$ in univariate analysis (Table 5).

Decreased counts of CD8 (OR 0.99; 95\% CI 0.98$1.00)$ and TLR2 (rs5743708) (G/G) (OR 23.10; 95\% CI 3.57-149.29) were associated with TLR2 (rs111200466) (Del/Del) in multivariate analysis (Table 6). A lower percentage of $\mathrm{CD} 8$, higher ratio of $\mathrm{CD} 4$ to $\mathrm{CD} 8$, and increased initial DIC onset by JAAM criteria were associated with TLR2 (rs111200466) (Del/Del) in the univariate analysis (Table 6).

\section{Discussion}

In the present study, we evaluated whether polymorphic variants of TLR2 and TLR4 are associated with 
Table 3 Prognostic Factors for Initial DIC Onset by JAAM Criteria

\begin{tabular}{|c|c|c|c|c|}
\hline Factors & No & Yes & $\begin{array}{c}\text { Univariate } \\
\text { Analysis OR } \\
(95 \% \mathrm{Cl})\end{array}$ & $\begin{array}{c}\text { Multivariate } \\
\text { Analysis OR } \\
(95 \% \mathrm{CI})^{*}\end{array}$ \\
\hline \multicolumn{5}{|l|}{ Shock } \\
\hline No & $117(82.98)$ & $22(55.00)$ & 1.00 & \\
\hline Yes & $24(17.02)$ & $18(45.00)$ & $3.99(1.86,8.55)$ & \\
\hline \multicolumn{5}{|l|}{ Death } \\
\hline No & $122(86.52)$ & $27(67.50)$ & 1.00 & \\
\hline Yes & $19(13.48)$ & $13(32.50)$ & $3.09(1.36,7.02)$ & \\
\hline \multicolumn{5}{|l|}{$\begin{array}{l}\text { Platelet count on admission }(145 \sim 400 \\
\left.\times 10^{9} / \mathrm{L}\right)\end{array}$} \\
\hline$<50$ & & $5(12.50)$ & $\begin{array}{c}23292468 \\
(0.00,1)\end{array}$ & \\
\hline $50-100$ & $16(11.35)$ & $25(62.50)$ & $\begin{array}{c}21.88 \\
(7.26,65.92)\end{array}$ & \\
\hline $100-150$ & $55(39.01)$ & $5(12.50)$ & $1.27(0.35,4.62)$ & \\
\hline$>150$ & $70(49.65)$ & $5(12.50)$ & 1.00 & \\
\hline Neutrophil percentage max (\%), mean \pm SD & $86.86 \pm 8.22$ & $90.33 \pm 5.51$ & $1.07(1.01,1.14)$ & \\
\hline \multicolumn{5}{|l|}{ Platelet count minimum $\left(145 \sim 400 \times 10^{9} / \mathrm{L}\right)$} \\
\hline$<50$ & $8(5.67)$ & $12(30.00)$ & $\begin{array}{c}20.25 \\
(5.23,78.38)\end{array}$ & \\
\hline $50-100$ & $23(16.3 \mid)$ & $21(52.50)$ & $\begin{array}{c}12.32 \\
(3.81,39.93)\end{array}$ & $\begin{array}{c}13.10 \\
(1.10,156.42)\end{array}$ \\
\hline $100-150$ & $56(39.72)$ & $3(7.50)$ & $0.72(0.15,3.38)$ & $1.55(0.10,23.19)$ \\
\hline$>150$ & $54(38.30)$ & $4(10.00)$ & 1.0000 & 1.00 \\
\hline$C D 3$, mean $\pm S D(|| 4|\sim| 880 / \mu L)$ & $614.31 \pm 341.86$ & $364.81 \pm 259.84$ & $1.00(0.99,1.00)$ & \\
\hline $\mathrm{CD} 4$, mean $\pm \mathrm{SD}(478 \sim 1072 / \mu \mathrm{L})$ & $371.69 \pm 230.98$ & $218.63 \pm 173.75$ & $1.00(0.99,1.00)$ & \\
\hline IL-2R on admission, mean $\pm \mathrm{SD}(\mathrm{U} / \mathrm{mL})$ & $1230.3 \pm 920.55$ & $2228.7 \pm 1172.4$ & $1.00(1.00,1.00)$ & \\
\hline \multicolumn{5}{|l|}{ IL-I0 on admission $(\mathrm{pg} / \mathrm{mL})$} \\
\hline$<9.1$ & $58(70.73)$ & $5(38.46)$ & 1.00 & \\
\hline$\geq 9.1$ & $24(29.27)$ & $8(61.54)$ & $3.87(1.15,13.02)$ & \\
\hline \multicolumn{5}{|l|}{ IFN- $\gamma$ on admission $(\mu g / L)$} \\
\hline$<5$ & $56(73.68)$ & $5(41.67)$ & 1.00 & \\
\hline$\geq 5$ & $20(26.32)$ & $7(58.33)$ & $3.92(1.12,13.77)$ & \\
\hline $\mathrm{CXCL8}$, mean $\pm \mathrm{SD}(\mathrm{pg} / \mathrm{mL})$ & $161.67 \pm 267.27$ & $411.49 \pm 438.64$ & $1.00(1.00,1.00)$ & \\
\hline $\mathrm{CXCL9}$, mean $\pm \mathrm{SD}(\mathrm{pg} / \mathrm{mL})$ & $58.75 \pm 87.85$ & $105.73 \pm 119.90$ & $1.00(1.00,1.01)$ & \\
\hline CXCLI0, mean \pm SD $(\mathrm{pg} / \mathrm{mL})$ & $4|8.8| \pm 650.14$ & $1117.4 \pm 2787.8$ & $1.00(1.00,1.00)$ & \\
\hline $\mathrm{CCL} 2$, mean $\pm \mathrm{SD}(\mathrm{pg} / \mathrm{mL})$ & $843.07 \pm 1150.9$ & $1863.5 \pm 1632.7$ & $1.00(1.00,1.00)$ & \\
\hline $\mathrm{CCL} 3$, mean $\pm \mathrm{SD}(\mathrm{pg} / \mathrm{mL})$ & $18.69 \pm 24.28$ & $41.42 \pm 51.68$ & $1.02(1.01,1.03)$ & \\
\hline $\mathrm{CCL} 4$, mean $\pm \mathrm{SD}(\mathrm{pg} / \mathrm{mL})$ & $21.49 \pm 36.20$ & $38.90 \pm 52.42$ & $1.01(1.00,1.02)$ & \\
\hline $\mathrm{CCL} 5$, mean $\pm \mathrm{SD}(\mathrm{pg} / \mathrm{mL})$ & $2283.4 \pm 1442.8$ & $1404.4 \pm 1018.6$ & $1.00(1.00,1.00)$ & \\
\hline $\mathrm{CCL} 20$, mean $\pm \mathrm{SD}(\mathrm{pg} / \mathrm{mL})$ & $|I 5.4| \pm 175.72$ & $224.96 \pm 219.28$ & $1.00(1.00,1.00)$ & \\
\hline Initial APACHE, mean $\pm S D$ & $13.20 \pm 7.79$ & $19.15 \pm 9.15$ & $1.08(1.04, I .13)$ & \\
\hline Initial SOFA, mean \pm SD & $4.65 \pm 2.84$ & $7.73 \pm 3.67$ & $1.31(1.17,1.47)$ & \\
\hline $\begin{array}{l}\text { Maximum DIC scores by JAAM criteria, } \\
\text { mean } \pm \text { SD }\end{array}$ & $2.21 \pm 1.94$ & $5.83 \pm 1.52$ & $2.31(1.79,2.98)$ & $1.72(1.15,2.58)$ \\
\hline $\begin{array}{l}\text { Maximum DIC scores by ISTH criteria, mean } \\
\pm \text { SD }\end{array}$ & $2.60 \pm 1.27$ & $4.45 \pm 1.26$ & $3.06(2.06,4.55)$ & \\
\hline
\end{tabular}

(Continued) 
Table 3 (Continued).

\begin{tabular}{|c|c|c|c|c|}
\hline Factors & No & Yes & $\begin{array}{c}\text { Univariate } \\
\text { Analysis OR } \\
(95 \% \mathrm{Cl})\end{array}$ & $\begin{array}{c}\text { Multivariate } \\
\text { Analysis OR } \\
(95 \% \mathrm{Cl}) *\end{array}$ \\
\hline $\begin{array}{l}\text { DIC onset by ISTH criteria } \\
\text { No } \\
\text { Yes }\end{array}$ & $\begin{array}{c}134(95.04) \\
7(4.96)\end{array}$ & $\begin{array}{l}27(67.50) \\
13(32.50)\end{array}$ & $\begin{array}{c}1.00 \\
9.22(3.37,25.24)\end{array}$ & \\
\hline $\begin{array}{l}\text { TLR2 (rs I I I 200466) } \\
\text {-/Del\&-/- } \\
\text { Del/Del }\end{array}$ & $\begin{array}{c}126(90.65) \\
13(9.35)\end{array}$ & $\begin{array}{c}30(76.92) \\
9(23.08)\end{array}$ & $\begin{array}{c}1.00 \\
2.91(1.14,7.43)\end{array}$ & \\
\hline $\begin{array}{l}\text { TLR4 (rsI I536889) } \\
\text { G/G } \\
\text { C/C\&C/G }\end{array}$ & $\begin{array}{l}89(64.03) \\
50(35.97)\end{array}$ & $\begin{array}{l}18(45.00) \\
22(55.00)\end{array}$ & $\begin{array}{c}1.00 \\
2.18(1.07,4.44)\end{array}$ & \\
\hline
\end{tabular}

Notes: *Univariate logistic regression and multivariate analyses were performed to determine the factors associated with initial DIC onset by JAAM criteria. A p value $<0.05$ was considered significant. Odds ratios (ORs) and their $95 \%$ confidence intervals were calculated.

thrombocytopenia and coagulation failure in patients with sepsis. We found that the genotypes of TLR2 (rs111200466) (Del/Del) and TLR4 (rs11536889) (C/ $\mathrm{C} \& \mathrm{C} / \mathrm{G}$ ) were associated with either initial DIC onset or DIC onset during hospitalization. DIC was associated with the minimum platelet count or admission platelet count, while enhanced activation of the cytokine and chemokine network, which predicted severe thrombocytopenia, was also associated with DIC in our study.

Platelets are well known for their role in hemostasis; they adhere to the damaged endothelium and participate in the formation of stable clots. In addition, platelets serve as potent amplifiers of the coagulation cascade. ${ }^{30}$ Platelets play a role in the development of DIC, a common complication observed in patients with sepsis. ${ }^{31}$ During DIC, platelets are activated and form smaller thrombi in the microvasculature or aggregates that are sequestered in organs such as the lungs. Together, this leads to thrombocytopenia, a reduction in the number of circulating platelets. ${ }^{13}$ Sepsis-associated thrombocytopenia has been recognized for many years and is a predictor of adverse outcomes. ${ }^{3}$ This might be a reasonable explanation for the association between lower plasma platelet count and higher risk of DIC, which is observed in our study.

Platelets utilize a highly regulated system for cytokine release. ${ }^{17}$ Platelets release chemokines of the $\mathrm{CXC}$ and $\mathrm{CC}$ classes, such as CXCL7 (NAP2), CXCL4 (PF4), CXCL1 (GRO-a), CXCL5 (ENA-78), CCL5 (RANTES), CCL3 (MIP-1a) and CCL7 (MCP-3), ${ }^{32}$ which are synthesized in megakaryocytes and stored in secretory vesicles, known as a-granules. ${ }^{33}$ CCL5 released by platelets in heterotypic aggregates works in concert with P-selectin on target leukocytes to induce the expression of cytokines and chemokines. ${ }^{34}$ CCL5 also associated with the surface of inflamed endothelial cells, providing localized signals for monocyte adhesion. ${ }^{35}$ Thus, platelet CCL5 can activate key innate immune effector cells and orchestrate immune interactions, and has the potential to activate T lymphocytes. ${ }^{35}$ CCL3 and CCL7 have similarly diverse activities. ${ }^{36}$ In addition to the lower platelet count associated with DIC, we observed lower CCL5 expression in patients with DIC. As with increased IL-8 and IL-10 levels in patients with platelets $<100 \times 10^{9} / \mathrm{L}$, mice depleted of platelets showed increased cytokine concentrations during endotoxemia and sepsis, ${ }^{9}$ and blood from platelet-depleted mice showed increased cytokine production in response to Klebsiella pneumonia infection. ${ }^{9}$ Interestingly, increased IL-8 and IL-10 were observed in patients with DIC in this study.

TLR4 is the most abundantly expressed TLR on platelets, ${ }^{37}$ and increasing evidence has indicated that the presence of TLR4 on platelets is a key regulator of the platelet number and function. ${ }^{15}$ Two studies ${ }^{38,39}$ showed that $\mathrm{TLR}^{-/-}$mice have defects in their circulating and reticulated platelet counts compared to wild-type mice, suggesting that TLR4 plays a role in thrombocytopoiesis. Mice stimulated by a non-lethal dose of a TLR4 ligand have a larger number of platelets compared to untreated mice. ${ }^{38}$ Platelets play an important role in affecting neutrophils and NET, with one of the key mediators being 
Table 4 Analysis of Potential Factors Predictive of Platelet Counts (100 versus $\geq 100)$

\begin{tabular}{|c|c|c|c|c|}
\hline Factors & $<100$ & $\geq 100$ & $\begin{array}{c}\text { Univariate } \\
\text { Analysis OR } \\
(95 \% \mathrm{Cl})\end{array}$ & $\begin{array}{c}\text { Multivariate } \\
\text { Analysis OR } \\
(95 \% \mathrm{CI})^{*}\end{array}$ \\
\hline \multicolumn{5}{|l|}{ Shock } \\
\hline No & $43(67.19)$ & $96(82.05)$ & 1.00 & \\
\hline Yes & $21(32.8 I)$ & $21(17.95)$ & $0.45(0.22,0.91)$ & \\
\hline WBC on admission, mean \pm SD $\left(4 \sim 10 \times 10^{9} / L\right)$ & $11.11 \pm 6.53$ & $14.26 \pm 7.17$ & $1.07(1.02,1.13)$ & $1.23(1.05,1.45)$ \\
\hline $\begin{array}{l}\text { Neutrophil on admission, mean } \pm \text { SD }(1.4 \sim 6.5 \\
\left.\times 10^{9} / \mathrm{L}\right)\end{array}$ & $9.93 \pm 6.11$ & $|2.46 \pm 6.7|$ & $1.07(1.01,1.12)$ & \\
\hline \multicolumn{5}{|l|}{ Platelet count on admission $\left(145 \sim 400 \times 10^{9} / \mathrm{L}\right)$} \\
\hline$<50$ & $5(7.81)$ & $0(0.00)$ & - & \\
\hline $50-100$ & 41 (64.06) & $0(0.00)$ & - & \\
\hline $100-150$ & $15(23.44)$ & $45(38.46)$ & $0.13(0.03,0.46)$ & \\
\hline$>150$ & $3(4.69)$ & $72(61.54)$ & 1.00 & \\
\hline$\% C D 4$, mean \pm SD & $33.85 \pm 12.43$ & $38.49 \pm 9.21$ & $1.05(1.00,1.09)$ & \\
\hline CD3, mean \pm SD $(|| 4|\sim| 880 / \mu L)$ & $418.15 \pm 299.62$ & $658.54 \pm 335.19$ & $1.00(1.00,1.00)$ & \\
\hline $\mathrm{CD} 4$, mean $\pm \mathrm{SD}(478 \sim 1072 / \mu \mathrm{L})$ & $234.42 \pm 191.99$ & $405.98 \pm 226.47$ & $1.00(1.00,1.01)$ & \\
\hline $\mathrm{IL}-2 \mathrm{R}$ on admission, mean $\pm \mathrm{SD}(\mathrm{U} / \mathrm{mL})$ & $2082.5 \pm 1170.3$ & $1020.3 \pm 709.03$ & $1.00(1.00,1.00)$ & $1.00(1.00,1.00)$ \\
\hline IL-6 on admission, mean $\pm \mathrm{SD}(\mathrm{pg} / \mathrm{mL})$ & $224.77 \pm 300.03$ & $87.16 \pm|66.4|$ & $1.00(1.00,1.00)$ & \\
\hline IL-8 on admission, mean \pm SD (pg/mL) & $383.52 \pm \mid 201.1$ & $34.70 \pm 28.37$ & $0.98(0.96,0.99)$ & $0.97(0.94,0.99)$ \\
\hline \multicolumn{5}{|l|}{ IL-I0 on admission $(\mathrm{pg} / \mathrm{mL})$} \\
\hline$<9.1$ & $12(38.7 \mathrm{I})$ & 51 (79.69) & 1.00 & \\
\hline$\geq 9.1$ & $19(61.29)$ & $13(20.3 \mid)$ & $0.16(0.06,0.41)$ & \\
\hline \multicolumn{5}{|l|}{ PAI-I (ng/mL) } \\
\hline$\leq 4363$ & 31 (48.44) & $75(64.10)$ & 1.00 & \\
\hline$>4363$ & $33(51.56)$ & $42(35.90)$ & $0.53(0.28,0.98)$ & \\
\hline $\mathrm{CXCL8}$, mean $\pm \mathrm{SD}(\mathrm{pg} / \mathrm{mL})$ & $290.43 \pm 380.80$ & $174.62 \pm 287.87$ & $1.00(1.00,1.00)$ & \\
\hline $\mathrm{CCL} 2$, mean $\pm \mathrm{SD}(\mathrm{pg} / \mathrm{mL})$ & $1533.3 \pm 1642.3$ & $805.31 \pm 1049.9$ & $1.00(1.00,1.00)$ & \\
\hline $\mathrm{CCL} 3$, mean $\pm \mathrm{SD}(\mathrm{pg} / \mathrm{mL})$ & $34.27 \pm 43.76$ & $17.73 \pm 24.30$ & $0.98(0.97,1.00)$ & \\
\hline $\mathrm{CCL} 5$, mean $\pm \mathrm{SD}(\mathrm{pg} / \mathrm{mL})$ & $1536.8 \pm 1036.5$ & $2399.9 \pm 1492.1$ & $1.00(1.00,1.00)$ & \\
\hline $\mathrm{CCL} 20$, mean $\pm \mathrm{SD}(\mathrm{pg} / \mathrm{mL})$ & $188.20 \pm 222.95$ & $112.08 \pm 165.40$ & $1.00(1.00,1.00)$ & $1.01(1.00,1.02)$ \\
\hline Initial SOFA, mean \pm SD & $6.66 \pm 3.60$ & $4.60 \pm 2.88$ & $0.82(0.75,0.91)$ & \\
\hline Initial DIC scores by JAAM criteria, mean \pm SD & $3.75 \pm 2.31$ & $1.30 \pm 1.23$ & $0.46(0.36,0.58)$ & \\
\hline \multicolumn{5}{|l|}{ Initial DIC onset by JAAM criteria } \\
\hline No & $31(48.44)$ & $110(94.02)$ & 1.00 & \\
\hline Yes & $33(5 \mathrm{I} .56)$ & $7(5.98)$ & $0.06(0.02,0.15)$ & \\
\hline $\begin{array}{l}\text { Maximum DIC scores by JAAM criteria, mean } \pm \\
\text { SD }\end{array}$ & $5.16 \pm 2.20$ & $1.83 \pm 1.50$ & $0.42(0.33,0.54)$ & \\
\hline \multicolumn{5}{|l|}{ DIC onset by JAAM criteria } \\
\hline No & $14(2 \mid .88)$ & $97(82.91)$ & 1.00 & \\
\hline Yes & $50(78.13)$ & $20(17.09)$ & $0.06(0.03,0.12)$ & \\
\hline $\begin{array}{l}\text { Maximum DIC scores by ISTH criteria, mean } \pm \\
\text { SD }\end{array}$ & $4.22 \pm 1.41$ & $2.34 \pm 1.04$ & $0.20(0.12,0.33)$ & $0.13(0.04,0.44)$ \\
\hline \multicolumn{5}{|l|}{ DIC onset by ISTH criteria } \\
\hline No & $46(71.88)$ & 115 (98.29) & 1.00 & \\
\hline Yes & $18(28.13)$ & $2(I .7 I)$ & $0.04(0.01,0.20)$ & \\
\hline
\end{tabular}

(Continued) 
Table 4 (Continued).

\begin{tabular}{|l|c|c|c|c|}
\hline Factors & $<100$ & $\geq 100$ & $\begin{array}{c}\text { Univariate } \\
\text { Analysis OR } \\
\mathbf{9} \text { Or\% Cl) }\end{array}$ & $\begin{array}{c}\text { Multivariate } \\
\text { Analysis OR } \\
\mathbf{( 9 5 \% ~ C l ) *}\end{array}$ \\
\hline TLR4 (rsII536889) & $29(45.31)$ & $78(67.83)$ & 1.00 & \\
G/G & $35(54.69)$ & $37(32.17)$ & $0.39(0.21,0.74)$ \\
C/C\&C/G & & & \\
\hline
\end{tabular}

Notes: *Univariate logistic regression and multivariate analyses were performed to determine the factors associated with platelet counts. A p value $<0.05$ was considered significant. Odds ratios (ORs) and their $95 \%$ confidence intervals were calculated.

TLR4, as it enables platelets to detect pathogen-associated molecular signatures circulating in the blood. During sepsis, TLR4 causes platelet binding to adherent NET formation in liver sinusoids and pulmonary capillaries, which facilitates bacterial capture. ${ }^{40}$ Platelets express functional
TLR4 that recognizes LPS, a major component of the cell wall of many Gram-negative bacteria. LPS, even at high concentrations, did not induce NET formation directly from neutrophils, suggesting that platelets are necessary for rapid LPS-induced neutrophil NET formation. ${ }^{40}$

Table 5 Analysis of Potential Factors Associated with Different Genotype of TLR4 (rs I I536889)

\begin{tabular}{|c|c|c|c|c|}
\hline Factors & G/G & $\mathbf{C} / \mathbf{C} \& \mathbf{C} / \mathbf{G}$ & $\begin{array}{c}\text { Univariate Analysis } \\
\text { OR }(95 \% \mathrm{Cl})\end{array}$ & $\begin{array}{c}\text { Multivariate Analysis } \\
\text { OR }(95 \% \mathrm{Cl})^{*}\end{array}$ \\
\hline \multicolumn{5}{|l|}{ Platelet counts on admission $\left(145 \sim 400 \times 10^{9} / \mathrm{L}\right)$} \\
\hline$<50$ & I (0.93) & $4(5.56)$ & $7.84(0.83,73.91)$ & $6.71(0.71,63.42)$ \\
\hline $50-100$ & $17(15.89)$ & $24(33.33)$ & $2.77(1.26,6.07)$ & $2.93(1.29,6.66)$ \\
\hline $100-150$ & $40(37.38)$ & $19(26.39)$ & $0.93(0.50,1.93)$ & $0.90(0.43,1.88)$ \\
\hline$>150$ & 49 (45.79) & $25(34.72)$ & 1.00 & 1.00 \\
\hline \multicolumn{5}{|l|}{ Platelet counts, minimum $\left(145 \sim 400 \times 10^{9} / \mathrm{L}\right)$} \\
\hline$<50$ & $8(7.48)$ & $12(16.67)$ & $3.00(1.05,8.58)$ & \\
\hline $50-100$ & $21(19.63)$ & $23(31.94)$ & $2.19(0.98,4.92)$ & \\
\hline $100-150$ & $40(37.38)$ & $18(25.00)$ & $0.90(0.41,1.97)$ & \\
\hline$>150$ & $38(35.5 \mathrm{I})$ & $19(26.39)$ & 1.00 & \\
\hline Initial DIC scores by JAAM criteria, mean \pm SD & $1.90 \pm 1.90$ & $2.58 \pm 2.23$ & $1.18(1.01,1.36)$ & \\
\hline \multicolumn{5}{|l|}{ Initial DIC onset by JAAM criteria } \\
\hline No & $89(83.18)$ & $50(69.44)$ & 1.00 & \\
\hline Yes & $18(16.82)$ & $22(30.56)$ & $2.18(1.07,4.44)$ & \\
\hline Maximum DIC scores by JAAM criteria, mean \pm SD & $2.67 \pm 2.28$ & $3.54 \pm 2.48$ & $1.17(1.03,1.32)$ & \\
\hline \multicolumn{5}{|l|}{ DIC onset by JAAM criteria } \\
\hline No & $73(68.22)$ & $36(50.00)$ & 1.00 & \\
\hline Yes & $34(31.78)$ & $36(50.00)$ & $2.15(1.16,3.97)$ & \\
\hline Maximum DIC scores by ISTH criteria, mean \pm SD & $2.77 \pm 1.35$ & $3.39 \pm 1.61$ & $1.34(1.08,1.66)$ & \\
\hline \multicolumn{5}{|l|}{ DIC onset by ISTH criteria } \\
\hline No & $101(94.39)$ & $58(80.56)$ & 1.00 & \\
\hline Yes & $6(5.6 I)$ & $14(19.44)$ & $4.06(1.48,11.15)$ & \\
\hline \multicolumn{5}{|l|}{ TLR4 (rs7869402) $(85 \mathrm{bpC} / \mathrm{T})$} \\
\hline No & 91 (85.05) & 71 (98.6I) & 1.00 & 1.00 \\
\hline Yes & $16(14.95)$ & I (I.39) & $0.08(0.01,0.62)$ & $0.08(0.01,0.60)$ \\
\hline
\end{tabular}

Notes: *Univariate logistic regression and multivariate analyses were performed to determine the factors associated with different genotype of TLR4 (rs I I536889). A $p$ value $<0.05$ was considered significant. Odds ratios (ORs) and their $95 \%$ confidence intervals were calculated. 
Table 6 Analysis of Potential Factors Associated with Different Genotype of TLR2 (rs I I I 200466)

\begin{tabular}{|c|c|c|c|c|}
\hline Factors & -/Del\&-/- & Del/Del & $\begin{array}{c}\text { Univariate } \\
\text { Analysis OR (95\% } \\
\text { Cl) }\end{array}$ & $\begin{array}{c}\text { Multivariate } \\
\text { Analysis OR (95\% } \\
\text { CI)* }\end{array}$ \\
\hline$\% C D 8$, mean $\pm S D$ & $22.63 \pm 8.79$ & $|6.42 \pm 6.4|$ & $0.90(0.82,0.99)$ & \\
\hline CD4/CD8, mean $\pm S D$ & $1.96 \pm 1.23$ & $3.54 \pm 2.84$ & $1.55(1.12,2.13)$ & \\
\hline $\mathrm{CD} 8$, mean $\pm \mathrm{SD}(393 \sim 742 / \mu \mathrm{L})$ & $215.48 \pm 146.20$ & $|18.4| \pm 53.55$ & $0.99(0.99,1.00)$ & $0.99(0.98,1.00)$ \\
\hline \multicolumn{5}{|l|}{ Initial DIC onset by JAAM criteria } \\
\hline No & $126(80.77)$ & $13(59.09)$ & 1.00 & \\
\hline Yes & $30(19.23)$ & $9(40.91)$ & $2.91(1.14,7.43)$ & \\
\hline \multicolumn{5}{|l|}{ TLR2 (rs5743708) } \\
\hline $\mathrm{A} / \mathrm{A}$ & 81 (52.26) & $5(22.73)$ & 1.00 & 1.00 \\
\hline$A / G$ & $65(41.94)$ & $4(18.18)$ & $1.00(0.26,3.86)$ & $0.93(0.16,5.29)$ \\
\hline $\mathrm{G} / \mathrm{G}$ & $9(5.8 \mathrm{I})$ & $13(59.09)$ & $23.40(6.77,80.88)$ & $23.10(3.57,149.29)$ \\
\hline
\end{tabular}

Notes: *Univariate logistic regression and multivariate analyses were performed to determine the factors associated with different genotype of TLR2 (rs I I I200466). A $P$ value $<0.05$ was considered significant. Odds ratios (ORs) and their $95 \%$ confidence intervals were calculated.

The functionality of TLR2 has also been studied in platelets, but not as extensively as that of TLR4. TLR2 is a highly inflammatory receptor that can recognize a very large number of PAMPs. ${ }^{17}$ Among these ligands are, inter alia, bacterial lipopeptides, peptidoglycan, and lipoteichoic acid from Gram-positive bacteria, micro-bacterial lipoarabinomannan, yeast zymosan, and even viral hemagglutinin. ${ }^{41}$ Platelet TLR2 is involved in histoneinduced thrombin generation during sepsis as histones are released from several apoptotic cells. ${ }^{17}$ The expression pattern of TLRs on platelets is unique in terms of the function and role of these cells in the pathogenesis of inflammatory diseases including sepsis and other chronic inflammatory disorders. ${ }^{42}$ Unlike TLR4, studies showed that stimulation of platelet TLR2 can directly activate platelets. Activation of this receptor with Pam3CSKKKK, a synthetic TLR2 ligand, results in platelet aggregation and adhesion. ${ }^{43}$

Most TLR members are also expressed on megakaryocytes, thus infection can modulate thrombopoiesis by affecting platelet production and function with enhanced GPIb and COX-2 expression via TLR2. ${ }^{44}$ In addition, stimulated megakaryocytes through TLR4 resulted in enhanced P-selectin (SELP) expression in pre-activated platelets under sepsis. ${ }^{45}$ Recently, ITGA2B expression was upregulated in circulating sepsis platelets via TLR4 showing a dynamic trafficking of specific RNA from megakaryocytes, and this was accompanied with increased production of integrin subunit IIb and activation of integrin IIb3, ${ }^{46}$ etc.
Previous studies have shown conflicting results regarding the effect of TLR2 and TLR4 SNP on sepsis. ${ }^{47,48}$ Additionally, no significant association between TLR2 polymorphisms (rs5743708, rs5743704, rs3804099) and sepsis was detected. ${ }^{48}$ Rodriguez-Osorio et al also found no association between TLR4 polymorphisms (rs4986790, rs4986791) and sepsis in a Mexican-Mestizo population. ${ }^{49}$ However, TLR2 SNP rs5743708 and TLR4 SNP rs4986790 were shown to be directly associated with severe sepsis or septic shock in another study. ${ }^{47}$ Two common SNPs exist in human TLR4, D299G and T399I, both of which are on the extra-cellular domain and are thought to reduce the effectiveness of endotoxin binding and, consequently, decrease the sensitivity to endotoxin. ${ }^{50}$ Sato et $\mathrm{al}^{51}$ found that the $\mathrm{G}$ allele of rs11536889 may suppress expression of TLR 4 by inhibiting translation rather than gene transcription. People with the G allele of rs11536889 show lower susceptibility to Gram-negative infections and better outcomes among patients with sepsis. ${ }^{52}$ The regulatory TLR4 polymorphism rs11536889 is associated with renal, coagulation, and hepatic organ failure in patients with sepsis. $^{52}$

In our study, TLR2 SNP (rs111200466) was significantly related to initial DIC onset as defined by JAAM criteria $(p<0.05)$. The Del/Del genotype greatly reduced initial DIC onset compared to that of the -/Del\&-/- genotype. Similarly, the genotype G/G of TLR4 (rs11536889) reduced the incidence of DIC compared to that of the genotype $\mathrm{C} / \mathrm{C} \& \mathrm{C} / \mathrm{G}$ in terms of initial DIC and DIC onset during hospitalization. Thus, the $\mathrm{G}$ allele of TLR4 
(rs11536889) up-regulated expression of TLR4 in platelets, which may have increased the activity of platelets $(>100 \times$ $\left.10^{9} / \mathrm{L}\right)$ and decreased incidence of thrombocytopenia. This activated or suppressed a series of cytokines and chemokines (IL-2R, IL-6, IL-8, IL-10, PAI-1, CCL2, CCL3, CCL5, and CCL20). Finally, the possibility of DIC was reduced. Although the data for TLR2 (rs111200466) was limited, we predict that rs111200466 changed the activity of TLR2 and weakened platelet activity, affecting the progression of DIC.

In conclusion, our results indicate that TLR2 (rs111200466) and TLR4 (rs11536889) variants, particularly TLR4 (rs11536889), are prognostic factors for DIC, which causes severe organ dysfunction and high mortality. TLR4 (rs11536889) polymorphisms are also associated with lower platelet counts that may predict the severity of inflammation.

\section{Acknowledgments}

We are most grateful to participants who generously agreed to provide samples for this study. The authors are also grateful to thank Dr. Hong-Hua Mu, Li Guo, Connor Meaney and Timothy Evan Moore from the University of Utah, Salt Lake City, United States of America, for helping us thoroughly review the manuscript.

\section{Author Contributions}

All authors made a significant contribution to the work reported, whether that is in the conception, study design, execution, acquisition of data, analysis and interpretation, or in all these areas; took part in drafting, revising or critically reviewing the article; gave final approval of the version to be published; have agreed on the journal to which the article has been submitted; and agree to be accountable for all aspects of the work.

\section{Funding}

This work was supported by the National Natural Science Foundation of China (No. 82172138, No.81772111 and No. 81873947), the Scientific Research and Innovation Program of Shanghai Municipal Science and Technology Commission (No. 21Y11902400), the New HundredTalent Program of Shanghai (No. 2018BR13), the Shanghai Shenkang Hospital Development Center (No. SHDC12016126), and the Key Laboratory of Emergency and Trauma (Hainan Medical University), Ministry of Education (Grant. KLET-202016).

\section{Disclosure}

The authors declare that they have no conflicts of interest.

\section{References}

1. Singer M, Deutschman CS, Seymour CW, et al. The third international consensus definitions for sepsis and septic shock (Sepsis-3). JAMA. 2016;315(8):801-810. doi:10.1001/jama.2016.0287

2. Huang M, Cai S, Su J. The pathogenesis of sepsis and potential therapeutic targets. Int J Mol Sci. 2019;20(21):5376. doi:10.3390/ ijms 20215376

3. Guo L, Rondina MT. The era of thromboinflammation: platelets are dynamic sensors and effector cells during infectious diseases. Front Immunol. 2019;10:2204. doi:10.3389/fimmu.2019.02204

4. Claushuis TA, van Vught LA, Scicluna BP, et al. Thrombocytopenia is associated with a dysregulated host response in critically ill sepsis patients. Blood. 2016;127(24):3062-3072. doi:10.1182/blood-201511-680744

5. Manne BK, Denorme F, Middleton EA, et al. Platelet gene expression and function in patients with COVID-19. Blood. 2020;136 (11):1317-1329. doi:10.1182/blood.2020007214

6. Yang X, Yang Q, Wang Y, et al. Thrombocytopenia and its association with mortality in patients with COVID-19. J Thromb Haemost. 2020;18(6):1469-1472. doi:10.1111/jth.14848

7. Rondina MT, Carlisle M, Fraughton T, et al. Platelet-monocyte aggregate formation and mortality risk in older patients with severe sepsis and septic shock. J Gerontol A Biol Sci Med Sci. 2015;70 (2):225-231. doi:10.1093/gerona/glu082

8. van den Boogaard FE, Schouten M, de Stoppelaar SF, et al. Thrombocytopenia impairs host defense during murine Streptococcus pneumoniae pneumonia. Crit Care Med. 2015;43(3): e75-e83. doi:10.1097/CCM.0000000000000853

9. de Stoppelaar SF, van 't Veer C, Claushuis TA, et al. Thrombocytopenia Impairs Host Defense in Gram-Negative Pneumonia-Derived Sepsis in Mice. Blood. 2014;124 (25):3781-3790.

10. Hottz ED, Bozza FA, Bozza PT. Platelets in immune response to virus and immunopathology of viral infections. Front Med. 2018;5:121. doi:10.3389/fmed.2018.00121

11. Carestia A, Kaufman T, Schattner M. Platelets: new bricks in the building of neutrophil extracellular traps. Front Immunol. 2016;7:271. doi:10.3389/fimmu.2016.00271

12. Levi M, Schultz M, van der Poll T. Sepsis and thrombosis. Semin Thromb Hemost. 2013;39(5):559-566. doi:10.1055/s-0033-1343894

13. Vallance TM, Zeuner MT, Williams HF, Widera D, Vaiyapuri S. Tolllike receptor 4 signalling and its impact on platelet function, thrombosis, and haemostasis. Mediators Inflamm. 2017;2017:9605894. doi: $10.1155 / 2017 / 9605894$

14. Trugilho MRO, Hottz ED, Brunoro GVF, et al. Platelet proteome reveals novel pathways of platelet activation and platelet-mediated immunoregulation in dengue. PLoS Pathog. 2017;13(5):e1006385. doi:10.1371/journal.ppat.1006385

15. Ding N, Chen G, Hoffman R, et al. Toll-like receptor 4 regulates platelet function and contributes to coagulation abnormality and organ injury in hemorrhagic shock and resuscitation. Circ Cardiovasc Genet. 2014;7(5):615-624. doi:10.1161/ CIRCGENETICS.113.000398

16. Kapur R, Semple JW. The nonhemostatic immune functions of platelets. Semin Hematol. 2016;53(Suppl 1):S2-S6. doi:10.1053/j. seminhematol.2016.04.002

17. Cognasse F, Nguyen KA, Damien P, et al. The inflammatory role of platelets via their TLRs and siglec receptors. Front Immunol. 2015;6:83. doi:10.3389/fimmu.2015.00083 
18. Aslam R, Speck ER, Kim M, et al. Platelet Toll-like receptor expression modulates lipopolysaccharide-induced thrombocytopenia and tumor necrosis factor- $\alpha$ production in vivo. Blood. 2006;107 (2):637-641. doi:10.1182/blood-2005-06-2202

19. Funayama H, Huang L, Sato T, et al. Pharmacological characterization of anaphylaxis-like shock responses induced in mice by mannan and lipopolysaccharide. Int Immunopharmacol. 2009;9(1314):1518-1524. doi:10.1016/j.intimp.2009.09.006

20. Mukherjee S, Huda S, Sinha Babu S. Toll-like receptor polymorphism in host immune response to infectious diseases: a review. Scand J Immunol. 2019;90(1):e12771. doi:10.1111/sji.12771

21. Joosten LA, Abdollahi-Roodsaz S, Dinarello CA, O'Neill L, Netea MG. Toll-like receptors and chronic inflammation in rheumatic diseases: new developments. Nat Rev Rheumatol. 2016;12 (6):344-357. doi:10.1038/nrrheum.2016.61

22. Schmitt C, Humeny A, Becker CM, Brune K, Pahl A. Polymorphisms of TLR4: rapid genotyping and reduced response to lipopolysaccharide of TLR4 mutant alleles. Clin Chem. 2002;48 (10):1661-1667. doi:10.1093/clinchem/48.10.1661

23. Shashkin PN, Brown GT, Ghosh A, Marathe GK, McIntyre TM. Lipopolysaccharide is a direct agonist for platelet RNA splicing. $J$ Immunol. 2008;181(5):3495-3502. doi:10.4049/jimmunol. 181.5.3495

24. Patrignani P, Di Febbo C, Tacconelli S, et al. Reduced thromboxane biosynthesis in carriers of toll-like receptor 4 polymorphisms in vivo. Blood. 2006;107(9):3572-3574. doi:10.1182/blood-2005$12-4811$

25. Gando S, Iba T, Eguchi Y, et al. A multicenter, prospective validation of disseminated intravascular coagulation diagnostic criteria for critically ill patients: comparing current criteria. Crit Care Med. 2006;34(3):625-631. doi:10.1097/01.CCM.0000202209.42491.38

26. Taylor FB, Toh CH, Hoots WK, Wada H, Levi M. Towards definition, clinical and laboratory criteria, and a scoring system for disseminated intravascular coagulation. Thromb Haemost. 2001;86 (5):1327-1330. doi:10.1055/s-0037-1616068

27. Vincent JL, de Mendonca A, Cantraine F, et al. Use of the SOFA score to assess the incidence of organ dysfunction/failure in intensive care units: results of a multicenter, prospective study.. Crit Care Med. 1998;26(11):1793-1800.

28. Knaus WA, Draper EA, Wagner DP, Zimmerman JE. APACHE II a severity of disease classification system. Crit Care Med. 1985;13 (10):818-829. doi:10.1097/00003246-198510000-00009

29. Huang Z, Fu Z, Huang W, Huang K. Prognostic value of neutrophil-to-lymphocyte ratio in sepsis: a meta-analysis. $\mathrm{Am}$ J Emerg Med. 2020;38(3):641-647. doi:10.1016/j.ajem.2019.10.023

30. Engelmann B, Massberg S. Thrombosis as an intravascular effector of innate immunity. Nat Rev Immunol. 2013;13(1):34-45. doi: $10.1038 /$ nri3345

31. Levi M, van der Poll T. Coagulation and sepsis. Thromb Res. 2017;149:38-44. doi:10.1016/j.thromres.2016.11.007

32. Weyrich AS, Zimmerman GA. Platelets: signaling cells in the immune continuum. Trends Immunol. 2004;25(9):489-495. doi:10.1016/j.it.2004.07.003

33. Romagnani P, Lasagni L, Annunziato F, Serio M, Romagnani S. CXC chemokines: the regulatory link between inflammation and angiogenesis. Trends Immunol. 2004;25(4):201-209. doi:10.1016/j. it.2004.02.006

34. McIntyre TM, Prescott SM, Weyrich AS, Zimmerman GA. Cell-cell interactions: leukocyte-endothelial interactions. Curr Opin Hematol. 2003;10(2):150-158. doi:10.1097/00062752-200303000-00009

35. Schober A, Manka D, von Hundelshausen P, et al. Deposition of platelet RANTES triggering monocyte recruitment requires P-selectin and is involved in neointima formation after arterial injury. Circulation. 2002;106(12):1523-1529. doi:10.1161/01.CIR.0000028590.02477.6F
36. Boehlen F, Clemetson KJ. Platelet chemokines and their receptors: what is their relevance to platelet storage and transfusion practice? Transfus Med. 2001;11(6):403-417. doi:10.1046/j.13653148.2001.00340.x

37. Berthet J, Damien P, Hamzeh-Cognasse H, Pozzetto B, Garraud O, Cognasse $\mathrm{F}$. Toll-like receptor 4 signal transduction in platelets: novel pathways. Br J Haematol. 2010;151(1):89-92. doi:10.1111/j.13652141.2010.08292.x

38. Jayachandran M, Brunn GJ, Karnicki K, Miller RS, Owen WG, Miller VM. In vivo effects of lipopolysaccharide and TLR4 on platelet production and activity: implications for thrombotic risk. J Appl Physiol. 2007;102(1):429-433. doi:10.1152/ japplphysiol.01576.2005

39. Andonegui G, Kerfoot SM, McNagny K, Ebbert KV, Patel KD, Kubes P. Platelets express functional Toll-like receptor-4. Blood. 2005;106(7):2417-2423. doi:10.1182/blood-2005-03-0916

40. Clark SR, Ma AC, Tavener SA, et al. Platelet TLR4 activates neutrophil extracellular traps to ensnare bacteria in septic blood. Nat Med. 2007;13(4):463-469. doi:10.1038/nm1565

41. Kawai T, Akira S. The role of pattern-recognition receptors in innate immunity: update on Toll-like receptors. Nat Immunol. 2010;11 (5):373-384. doi:10.1038/ni.1863

42. Vijay K. Toll-like receptors in immunity and inflammatory diseases: past, present, and future. Int Immunopharmacol. 2018;59:391-412. doi:10.1016/j.intimp.2018.03.002

43. Blair P, Rex S, Vitseva O, et al. Stimulation of Toll-like receptor 2 in human platelets induces a thromboinflammatory response through activation of phosphoinositide 3-kinase. Circ Res. 2009;104 (3):346-354.

44. Beaulieu LM, Lin E, Morin KM, Tanriverdi K, Freedman JE. Regulatory effects of TLR2 on megakaryocytic cell function. Blood. 2011;117(22):5963-5974. doi:10.1182/blood-2010-09-304949

45. Szilágyi B, Fejes Z, Póliska S, et al. Reduced miR-26b expression in megakaryocytes and platelets contributes to elevated level of platelet activation status in sepsis. Int $J$ Mol Sci. 2020;21(3):866. doi:10.3390/ijms21030866

46. Middleton EA, Rowley JW, Campbell RA, et al. Sepsis alters the transcriptional and translational landscape of human and murine platelets. Blood. 2019;134(12):911-923. doi:10.1182/ blood.2019000067

47. Gao JW, Zhang AQ, Wang X, et al. Association between the TLR2 Arg753Gln polymorphism and the risk of sepsis: a meta-analysis. Crit Care. 2015;19:416. doi:10.1186/s13054-015-1130-3

48. Smeland TE, Muller F, Blomfeldt A, Stavem K, Aamot HV. No associations established between single nucleotide polymorphisms in human Toll-like receptor 2 and Toll-interacting protein and Staphylococcus aureus bloodstream infections. APMIS 2017;125 (10):927-932.

49. Rodriguez-Osorio CA, Lima G, Herrera-Caceres JO, et al. Genetic variations in toll-like receptor 4 in Mexican-Mestizo patients with intra-abdominal infection and/or pneumonia. Immunol Lett. 2013;153 (1-2):41-46. doi:10.1016/j.imlet.2013.07.002

50. Rallabhandi P, Bell J, Boukhvalova MS, et al. Analysis of TLR4 polymorphic variants: new insights into TLR4/MD-2/CD14 stoichiometry, structure, and signaling. J Immunol. 2006;177(1):322-332. doi:10.4049/jimmunol.177.1.322

51. Sato K, Yoshimura A, Kaneko T, et al. A single nucleotide polymorphism in 3 -untranslated region contributes to the regulation of Toll-like receptor 4 translation. $J$ Biol Chem. 2012;287 (30):25163-25172. doi:10.1074/jbc.M111.338426

52. Mansur A, von Gruben L, Popov AF, et al. The regulatory toll-like receptor 4 genetic polymorphism rs 11536889 is associated with renal, coagulation and hepatic organ failure in sepsis patients. $J$ Transl Med. 2014;12:177. doi:10.1186/1479-5876-12-177 


\section{Publish your work in this journal}

The Journal of Inflammation Research is an international, peerreviewed open-access journal that welcomes laboratory and clinical findings on the molecular basis, cell biology and pharmacology of inflammation including original research, reviews, symposium reports, hypothesis formation and commentaries on: acute/chronic inflammation; mediators of inflammation; cellular processes; molecular mechanisms; pharmacology and novel anti-inflammatory drugs; clinical conditions involving inflammation. The manuscript management system is completely online and includes a very quick and fair peerreview system. Visit http://www.dovepress.com/testimonials.php to read real quotes from published authors. 